\section{PRIMER PLAN DE SANEAMIENTO DE MADRID/ESPAÑA}

\author{
M. a Teresa Solesio de la Presa, \\ Lcda. en Filosofía y Letras \\ (I.E.T.c.c.)
}

533-25

\section{CONDUCCION DE AGUAS RESIDUALES EN EL MADRID DE 1700}

A pesar de encontrarnos en el año 1700, Madrid carecia de una red de conducciones de aguas residuales. El grito de "agua va" era todavía la voz dominante en pleno siglo XVIII, cuando nuestra Capital presumia de gran Ciudad europea. El problema era candente y dificil de resolver.

Después de muchos estudios, se llega al convencimiento de llevar a la práctica el proyecto del Ingeniero Arquitecto Don José de Arce nombrado por el Real Consejo.
¿En qué consistía este proyecto? Consistía en "hacer" que las inmundicias de Madrid corriesen, directamente al Río Manzanares, a través de una serie de minas y minetas subterráneas.

Se partía de una mina general, Ilamada Real, que iba desde la Puerta del Sol, letra A, a los Caños del Peral, letra $\mathrm{K}$ y a ella desaguaban una serie de minas que comprendía el Madrid del siglo XVIII.

El proyecto constaba de 115 minas, todas ellas numeradas. (Ver plano).

La mina n. ${ }^{\circ} 1$ partía de la Calle de Alcalá, aproximadamente a la altura de la actual Calle de Peligros, e iba hasta la letra A, comienzo de la mina Real, en la Puerta del Sol.

La mina $n .^{\circ} 2$ comenzaba en la Calle Angosta de San Bernardo, hoy Calle de la Aduana, alcanzaba la mina $n .^{\circ} 3$ y desde alli continuaba a la Calle de la Montera, mina . $^{\circ} 4$ por donde bajaba a la Mina Real, en la Puerta del Sol, letra A.

La mina n. ${ }^{\circ} 5$ partía de la Calle de los Jardines y llegaba a la Calle de la Montera, mina n. ${ }^{\circ} 6$ y pasando por la mina $n .^{\circ} 4$ iba a la Mina Real en la Puerta del Sol, letra A.

La mina $n .^{\circ} 7$ tenía su principio en la Red de San Luis, seguía por la Calle de la Montera, recibiendo las minas núms. 6 y 4 y juntas desaguaban en la Mina Real, en la Puerta del Sol, letra A.

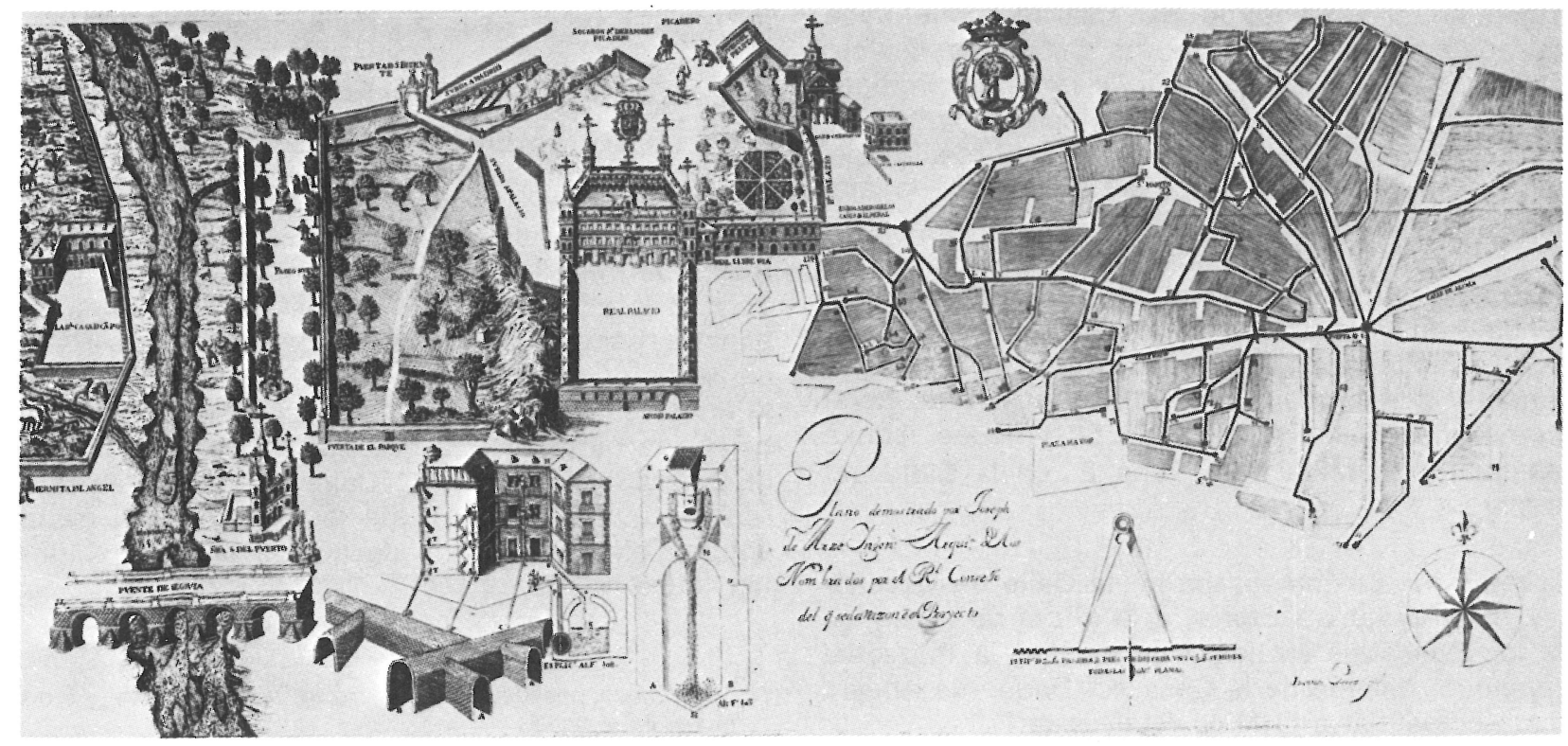


La mina $n .^{\circ} 8$ tenía su comienzo en la parte alta de la Calle Tres Cruces, recorria esta Calle y seguia a través del Cementerio de San Luis, hoy Plaza del Carmen, a la Calle de los Negros, en la actualidad Calle Tetuán; proseguía por la Calle del Carmen mina $n .^{\circ} 9$ y de aquí directamente, a la Mina Real, en la Puerta del Sol, letra A.

Las minas 10 y 11 hay que situarlas en las Calles de la Salud y de Chinchilla, las cuales se juntaban en la mina $n .{ }^{\circ} 12$ y bajaban hasta la Calle del Carmen para encontrar las minas núms. 13 y 9 y llegar a la Mina Real en la Puerta del Sol, letra $A$.

Las minas números 14 y 15 correspondian a las Calles Olivo Alta, hoy Calle de Mesonero Roma. nos y a la Calle Abada que se unian en la mina número 16, confluencia de Abada y Mesonero Romanos, para proseguir a la mina n.॰ 17 por la Calle del Carmen y de aqui junto con las minas 13 y 9 llegar a la Mina Real, letra $A$, en la Puerta del sol.

La mina $n 0^{\circ} 19$ partía de la Calle de Abada, recoría la de San Jacinto, hoy parte de la Plaza del Callao y doblando en la mina n. 18 llegaba a la Calle del Carmen, seguia a la Calle Rompelanzas $y$ de aqui a la mina $n x^{\circ} 20$ en la calle de Preciados; proseguia hasta la mina $n .^{\circ} 21$ en la Calle del Candil, hoy Galdo desaguando en la Mina Real, en la Puerta del Sol, letra 8.

La mina n. 22 estaba situada al comienzo del Postigo de San Martin, junto a las casas del Conde de Moriana y se unía a la mina $n .^{\circ} 23$ que bajaba por la Calle Preciados, rodeando las tapias del Convento de las Descalzas Reales; continuaba por Capellanes, hoy Maestro Victoria hasta la Mina número 24, que a su vez recibía la mineta de la Calle del Codo, hoy Callejón de Preciados, letra $X$.

Más abajo recibia la mina $n 0^{\circ} 25$ que tenía su origen en el Postigo de San Martín; continuaba doblando por la Tahona de las Descalzas Reales, mina n. 26 , para seguir por la calle de Peregrim nos, hoy parte de la calle de Tetuán, mina n. ${ }^{\circ} 27$ y doblar en la mina n. ${ }^{\circ} 28$, en la calle de la Zar. za. Esta parte de Calle hoy no existe por la ampliación de la Puerta del Sol.

Seguia la mina n.029 por la Calle del Arenal, donde empalmaba con otra mineta, que venia desde el principio de la Calle de la Zarza, hoy Calle Tetuán y bordeando el recodo de la Calle de Peregrinos encontraba la mina n. 30 y todas iban a desaguar a la Mina Real, letra $E$, en la Calle del Arenal.

La mina $n .^{\circ} 31$ comenzaba en la Calle de Capellanes, hoy Maestro Victoria, a la altura de la Botica de las Descalzas Reales y bajaba por la Plaza del Celenque, delante de la Casa del Duque de Abeyro hasta empalmar a la Mina Real, letra F.
La mina $n .^{\circ} 32$ partía de la Plazuela de San Martín junto a las Descalzas y por la calle de San Martín llegaba a la Mina Real, letra $G$, en el lugar conocido por Puertecilla de San Ginés.

La mina n. 33 comenzaba en la Plazuela de San Martín, bajaba por la Calle de Hileras desaguando en la Mina Real, letra $H$.

La mina n. ${ }^{\circ} 34$ partia de la Calle de la Sartén, hoy Navas de Tolosa y después de recibir las minetas de las Calle de la Ternera, mina $n .^{\circ} 35$, y de las Veneras, mina $n .^{\circ} 36$, continuaba por la Calle de la Sartén, hoy Calle de las Conchas, donde estaba situada la mina n. 37 ; bajaba por la Calle de la Rosa, hoy Costanilla de los Angeles, mina $n .{ }^{\circ} 38$ a la Plaza de Santa Catalina de los Donados, mina número 39 y de aqui a encontrar la Mina Real, letra L.

La mina n. 40 comenzaba en la confluencia de la Calle de la Sartén, hoy Conchas y Trujillos, bajaba por ésta, doblaba la calle de Santa Catalina la Vieja, hoy Flora, mina $n 0^{\circ} 41$ y por la Calle de Santa Catalina de los Donados, mina $n .^{\circ} 42$ des. aguaba en la Mina Real, letra $X$.

De nuevo y en el lugar de partida en la calle de Alcalá a la altura de la Calle de Peligros corría la mina n. 45 (1) que transcurria por la Calle de los Peligros, actual Calle de Sevilla, recibiendo a mitad de la misma, la mineta $n .^{\circ} 46$, que procedia de la Calle Bodegones. Esta calle hoy no existe; hay que situarla como medianera entre el Banco Español de Crédito y el Banco Hispano Americano de la Plaza de Canalejas. Continuaba hasta las Cuatro Calles, hoy Plaza de Canalejas, mina número 47 donde a su vez recibía la mina $n .^{\circ} 48$ que procedia de la Calle del Principe; más delante estaba la mina $n .{ }^{\circ} 49$ que arrancando de la esquina de la Calle del Pozo, bajaba por la Calle de la Cruz y por la Carrera de San Jerónimo desaguaba en la Mina Real, en la Puerta del Sol, letra A.

A la Carrera de San Gerónimo acudía la mina número 51, que arrancaba de la Calle de la Cruz a la altura de la hoy Calle de la Victoria, bajaba por la Calle de la Cruz, doblaba por la Calle del Pozo, mina $n .^{\circ} 52$, hasta la mina $n .^{\circ} 53$, que a su vez recibia la mina $n .^{\circ} 54$, que venia de la Calle de la Cruz, a la altura de la hoy Calle Espoz y Mina y, bajando por la Calle de la Cruz, llegaba a la Calle de la Victoria. Asimismo de la Calle de la Cruz, a la altura de las hoy Plazas del Angel y Benavente, bajaba la mina $n .^{\circ} 55$, que seguía por la Calle de la Cruz a la mina $n .^{\circ} 56$, Calle Ancha de Majaderitos, hoy Barcelona, hasta la mina n. 57 , en la actual Calle de Cádiz y de aquí a la Calle Carretas, mina n. ${ }^{\circ} 60$.

(1) Es curioso observar que las minas números 43 y 44 no figuran en el plano. 
Informes de la Construcción/347

Partiendo de la Plaza de Benavente corría la mina número 61, que bajaba por la Calle de Carretas, mina $n .^{\circ} 60$ desembocando en la Mina Real, en la Puerta del Sol, letra A.

La mina $n .^{\circ} 62$ partía de la Plaza de la Leña, hoy Calle de la Bolsa, seguia por la Callejuela de la Paz, mina n. ${ }^{\circ} 63$, hasta llegar a la Mina Real, letra $B$.

La mina 64 arrancaba de la Calle del correo y desaguaba en la Mina Real, letra $P$.

La mina $n .^{\circ} 65$ corria junta al Palacio de Santa Cruz y por la Calle de Esparteros, bajaba a la Iglesia de San Phelipe el Real desaguando en la Mina Real, letra K. Antes recibia la mineta $n .^{\circ} 66$, que salia de la Plazuela de San Estevan, hoy confluencia de la Calle de Pontejos con la de Conde de Plasencia, e lba hasta la mina n. ${ }^{\circ} 67$. Asimis mo recibia las minas que corrian por la parte alta de las calles de San Cristoval y Vicario Viejo, hoy tramo de la Calle Conde de Plasencia, entre las Calles de San Cristobal y Esparteros, minas números 68 y 69, para desaguar todas en la Calle de Esparteros.

A la mina $n 0^{\circ} 71$, que estaba situada frente a la Iglesia de San Phellpe el Real llegaba la mina número 72 que venía de la Calle de Vicario Viejo, hoy Conde Plasencia y continuaba a la mina número 73 en la Calle de San Cristoval y por la Calle postas volvía de nuevo a la mina $n .{ }^{\circ} 71$.

La mina $n .^{\circ} 74$ comenzaba en la Calle de Postas esquina a la Calle de Zaragoza, bajaba por Postas hasta la mina $n .{ }^{\circ} 75$, donde doblaba por la Calle San Cristoval hasta la mina $n .^{\circ} 76$ en la Calle Mayor.

En la Plaza de Santa Cruz estaba situada la mina número 77 que corria por la Calle de Zaragoza y de aqui a la Plaza Mayor, mina $n .^{\circ} 78$ y a la Calle de los Botoneros, hoy Felipe III, mina n. ${ }^{\circ} 79$ y por la Calle Mayor, mina n. 80 terminaba desaguando en la Mina Real, letra $Q$.

La mina n. 81 estaba situada en la Plaza Mayor, junto a la Calle de los Botoneros, hoy Felipe III y corría frente a la Casa de la Panaderia, mina número 82 y empalmaba con la mina $n .{ }^{\circ} 83$ y con la mina n. 84 , que venía a su vez de la Calle Nueva hoy Ciudad Rodrigo; juntas bajaban por la Calle de la Amargura, hoy Siete de Julio, hasta la Calle Mayor, mina n. 85 para doblar por la Calle Coloreros, mina $n .^{\circ} 86$, hasta la Plazuela de San Ginés, mina 87 , por la Calle Bordadores a la mina n. ${ }^{\circ} 88$ y hasta la Mina Real, en la Calle del Arenal, letra $G$.

La mina n. 89 partia de la Puerta de Guadalajara, confluencia de la Plaza de Comandante de las Morenas y Plaza Mayor, y por las Calles Mayor y Bordadores llegaba a la Mina Real, letra $G$.
La Mina n. 90 estaba situada en la confluencia de San Felipe Neri, Bordadores y Mayor, seguia por la Plaza de Herradores, mina n.0 91 y por Hileras, mina n. ${ }^{\circ} 92$, desaguaba en la Mina Real. letra $\mathrm{H}$.

La mina n. 93 arrancaba de la Calle de la Caza, hoy Plaza del Comandante de las Morenas, bajaba por la Plaza de Herradores y por la calle de las Fuentes, mina n. ${ }^{\circ} 94$, llegaba a la Calle del Are nal, a la Mina Real, letra $N$.

La mina $n .^{\circ} 95$ comenzaba en el primer tramo de la Calle Bonetillo, hoy Plaza del Comandante de las Morenas, bajaba por la actual Calle del Bonetillo hasta la Calle Tintoreros, donde recibia la mi. na n. 97 que venía de la Calle Mesón de Paños. La zona por donde corria esta mina habria que situarla hoy en la manzana entre las calles Co. mandante de las Morenas y Milaneses.

Aqui a su vez venia a parar la mina n.० 99, que desde Milaneses, Calle de Santiago lba por la Costanilla de Santiago. En este punto se encontraba la mina $n .^{\circ} 96$, que por la Calle Escalinata, mina n. 98 , iba a desembocar a los caños del Peral, letra $\mathrm{K}$.

La mina n. 100 arrancaba de la Calle del Espejo esquina a Santiago; continuaba hasta la mina número 101, donde recibia otra mina que venía de la Calle de Santa Clara, mina $n .^{\circ} 102 y$, a través de la Calle Conde de Lemos, bajaban juntas a la mina $n .{ }^{\circ} 103$, situada en la confluencia de Amnistia, Espejo, Lazo e Independencia. A esta mina, asimismo, concurrian la mina n. 104, que lba por la Calle del Lazo y la mina $n .^{\circ} 105$ que venia de la actual Calle de Amnistia entonces parte de Es. pejo para continuar a la mina $n .^{\circ} 106$, en la confluencla de las Calles Independencia y Vergara, y a la Calle de Santa Catalina la Vieja, mina n. ${ }^{\circ} 107$, hoy parte de la Calle Carlos III, y por la Calle Vergara $0^{\circ} 108$, llegaba a la Mina Real, letra K.

La mina $n .^{\circ} 109$ estaba situada en una Calle sin salida, hoy coincidente, en parte, con la Calle Conde Lemos, doblaba en la Plaza de Santiago mina $n .^{\circ} 110$ y bajaba por la Calle de Santa Clara mina n. 111, confluencia de Amnistía y Sta. Claw ra, doblando para unirse en la mina $n .^{\circ} 112$, con la que partia de la Calle de los Salvajes hoy San Nicolás mina n. ${ }^{\circ} 113$, seguía a la mina $n .^{\circ} 114$ en la Real Libreria de Palacio y de aqui pasaba a la mina $n .^{\circ} 115$, hoy Teatro Real, desembocando en la Mina Real, letra $K$, Caños del Peral.

Desde este punto de los Caños del Peral y a través de Palacio y los actuales Jardines de Sabatini, Campo del Moro, Paseo Nuevo, hoy Virgen del Puerto, llegaban al Río Manzanares, aguas abajo del actual Puente del Rey, las aguas residuales de Madrid. 


\section{RECONOCIMIENTO}

Mi más sincero agradecimiento a Carlos Rodríguez Ginestal por su inestimable colaboración.

\section{BIBLIOGRAFIA}

ARCE, José Alonso de

Dificultades vencidas y curso natural en que se dan reglas especulativas y prácticas para la limpieza de las calles de esta Corte, por cuyo medio se obvia que en el ambiente se introduzcan lo impuro... propone varias dificultades con un discurso sobre el gobierno entre Madrid y los dueños de las casas en la práctica de Limpieza. Madrid, F. Martínez Abad.
MOLINA CAMPUZANO, Miguel

El plano de Madrid por Texira, estampado en 1656.

Madrid. Artes Gráficas Municipales 1975.

PEÑASCO, H. y CAMBRONERO, C.

Las calles de Madrid. Noticias, tradiciones y curiosidades.

Ed. Facsímil. Madrid 1889. Madrid 1975.

\section{TEXEIRA, Pedro}

Topografía de la Villa de Madrid. Año 1656. Madrid 1977.

\section{publicaciones del I.E.T.C.C.}

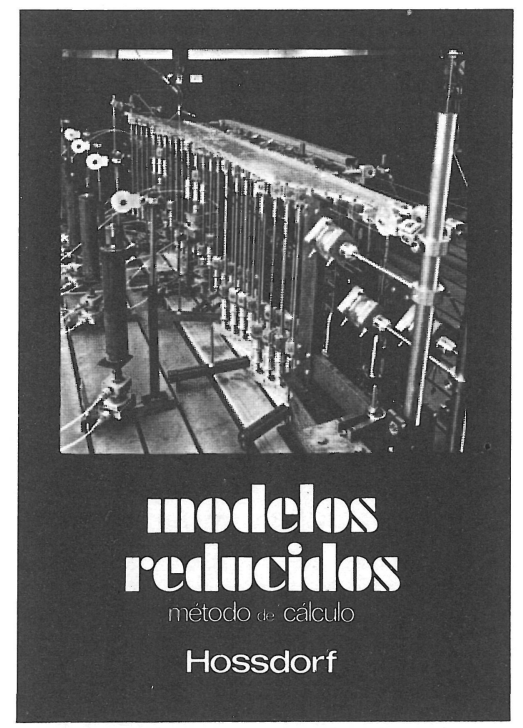

Modelos reducidos. Método de cálculo

H. Hossdorf, Ingeniero Civil

La técnica de los ensayos en modelos reducidos de estructuras sufre hoy dia una decisiva metamorfosis. Hasta hace poco era un medio más bien de artesania, que no siempre era tomado en serio por los académicos teorizantes para comprender el comportamiento resistente de las estructuras complejas y a que se acudió las más de las veces, como a un último remedio debido a sus indiscutibles insuficiencias. Sin embargo, en poco tiempo y gracias a su conexión con los ordenadores digitales, se ha transformado en un instrumento científicamente valioso, que no puede quedar a un lado en la práctica diaria del Ingeniero Proyectista.

Un volumen encuadernado en cartoné plastificado con lomo de tela, de $17 \times 24 \mathrm{~cm}$, compuesto de 250 páginas, 158 figuras $y$ fotografias.

Precios: 1.800 ptas.; \$ USA 26.00

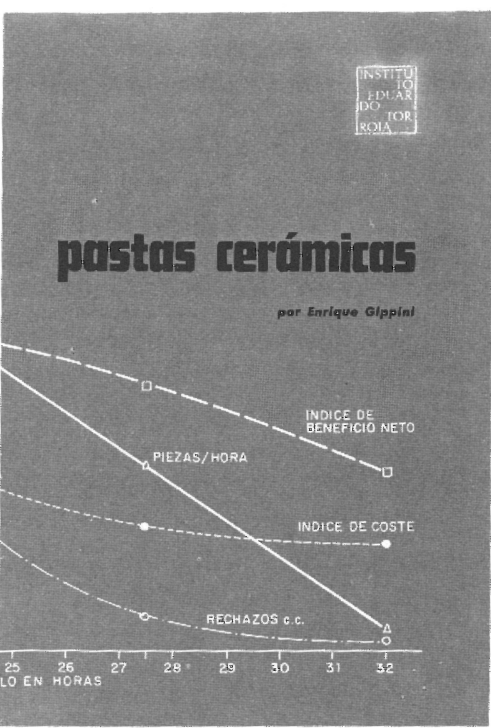

PASTAS CERAMICAS

Enrique Gippini,

Dr. en Ciencias Quimicas

El nexo de unión de todos los capítulos del libro es la idea subyacente de crear una teoria general de pastas. Moldeo y Cocción son los dos procesos a los que debe adecuarse la composición. Las características fisico-quimicas más importantes que deben presentar las pastas para que los resultados de estos procesos sean satisfactorios y cómo pueden cambiarse dichas caracteristicas son los temas de discusion
escogidos.

Un volumen encuadernado en cartoné, de $25 \times 17 \mathrm{~cm}$ compuesto de 259 páginas, 143 figuras y fotografias, y 37 tablas.

Precios: 2.000 ptas.; \$ USA 29.00

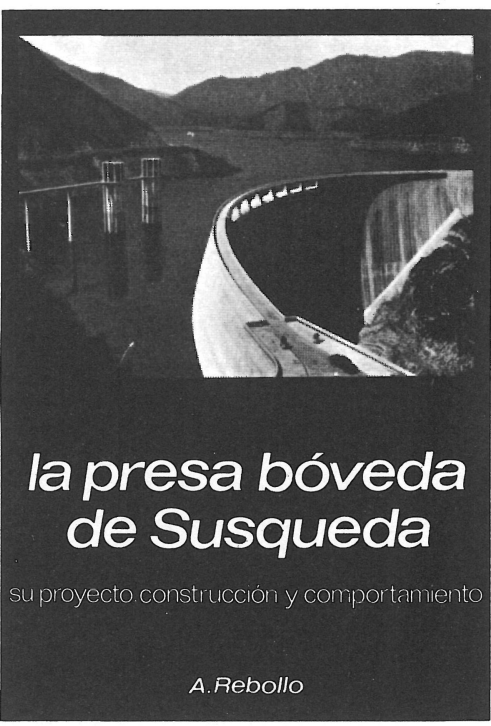

La presa bóveda de Susqueda

A. Rebollo,

Dr. Ingeniero de Caminos

El esfuerzo del constructor de presas se sitúa por su pretensión de perennidad, a contracorriente de las tendencias de la civilización actual, caracterizada por lo fungible. Pueden evocarse las 10.000 grandes. presas en funcionamiento o en construcción que están envejeciendo y reclaman los cuidados gerontológicos para mantener y perfeccionar su servicio y garantizar su inalienable pretensión de perennidad. En la medida en que todas nuevas obras, grandes o pequeñas, son portadoras de riesgos ecológicos $y_{1}$ a veces, catastróficos, que aumentan con el envejecimiento, la gerontologia de las presas es todo un emplazo. La accion adelantada de Arturo Rebollo en este terreno marca un camino levión pados los que aman su propia obra con Un volumen encuadernado en cartoné plastificado 408 páginas, 330 figuras y fotografias y 39 tablas. Precios: 1.700 ptas.; extranjero, \$ USA 24.00 . 